\title{
Estandarización del cuestionario para medir las habilidades gerenciales en el sector Turismo (HGT)
}

\section{Standardization of the questionnaire to measure the management skills in the tourism sector}

Irma Milagros Carhuancho Mendoza

Luis Guillermo Sicheri Monteverde

Fernando Alexis Nolazco Labajos

José Abel De la Torre Tejada

Universidad Norbert Wiener, Perú

Autor para correspondencia: irma.carhuancho@uwiener.edu.pe; luis.sicheri@uwiener.edu.pe; fernando.nolazco@uwiener.edu.pe; delatorretejada@yahoo.com

Fecha de recepción: 03 de junio de 2019 - Fecha de aceptación: 27 de agosto de 2019

Resumen: El estudio tuvo como objetivo establecer las dimensiones, calcular la confiabilidad y estandarizar el cuestionario de habilidades gerenciales en el sector turismo. Se seleccionaron directivos vinculados a la actividad turística predominando la participación del género masculino (54\%), las edades oscilaron entre 37 a 65 años. El cuestionario se sometió a la Prueba de KMO (.875) y Barlett ( $\mathrm{p}=.000)$, en la cuarta aplicación se logró obtener el valor aceptable; la prueba de Alfa de Cronbach $(\alpha=.968)$ y Dos mitades de Guttman $(\mathrm{G}=.918)$ demostraron que el cuestionario es confiable. Finalmente, el instrumento quedó conformado por 31 ítems, las dimensiones son: a) habilidades técnicas se mide a través de los indicadores software, idioma, negociación y decisiones; b) habilidades de trato personal, integrada por los indicadores autocontrol, autoconfianza, equilibrio emocional y empatía; c) habilidades conceptuales compuesta por el desarrollo de nuevos conceptos y resolución de problemas.

Palabras clave: habilidades gerenciales; habilidades técnicas; habilidades humanas; habilidades conceptuales; turismo

\begin{abstract}
The objective of the study was to establish the dimensions, calculate the reliability and standardize the management skills in a questionnaire for the tourism sector. We selected executives linked to the tourism activity predominating the participation of the male gender $(54 \%)$, the ages oscillated between 37 and 65 years. The questionnaire was tested for KMO (.875) and Barlett (P $=.000)$, in the fourth application the acceptable value was obtained; The Cronbach alpha Test $(\alpha$ $=.968)$ and two halves of Guttman $(\mathrm{G}=.918)$ showed that the questionnaire was reliable. At the end, the instrument was made up of 31 items and the dimensions were: a) technical skills measured through software, language, negotiation and decision indicators; B. Personal treatment skills, like self-control, self-confidence, emotional balance and empathy; C. Conceptual skills, composed of the development of new concepts and problem solving.
\end{abstract}

Key Words: management skills; technical skills; human skills; conceptual skills; tourism 


\section{Introducción}

Las organizaciones han cambiado en el trascurrir del tiempo; actualmente se requiere gerentes capacitados, especializados y sobre todo que posean habilidades. La habilidad es la capacidad que tiene el individuo para transformar el conocimiento en acción y lograr el resultado deseado (Maximiano, 2007), también es el conjunto de acciones o conductas, que sistematizan el actuar gerencial en una organización para alcanzar los objetivos personales y organizacionales (Katz, 1974, Reh, 2009, Rebolledo, Flores, Reyes y Flores, 2015, Lopes, y otros, 2010, Robbins y Coulter, 2005).

"Las habilidades pueden demostrarse, practicarse, mejorarse o frenarse conscientemente por los propios individuos [...] pueden implicar a otra gente y requieren trabajo cognoscitivo, pero son conductas que la gente puede controlar por sí misma” (Robbins y Coulter, 2005, p. 8), por consiguiente, las habilidades son aprendidas, modificadas, ayudan a controlar los comportamientos y permiten un mejor desenvolvimiento en la interrelación humana.

En este sentido las habilidades directivas son importantes para el desarrollo personal y profesional, en el siglo XXI se exige gerentes con flexibilidad cognitiva, liderazgo, inteligencia emocional, trabajo en equipo, comunicación, pensamiento crítico, valores éticos, capacidad de negociación y resolución de problemas (Ramírez, 2018; Reyes, 2015). Las habilidades directivas no son estáticas, se adaptan en el tiempo en relación a las transformaciones que se originan alrededor de la organización y los cambios estructurales.

Los gerentes en los últimos años se ven obligados a desempeñar con mayor firmeza sus habilidades gerenciales con el objetivo de rediseñar, innovar y crear nuevos productos para competir o simplemente dejar de existir. Un claro ejemplo es el caso de la incursión en el mercado el uso de plataformas como Airbnb y servicio de taxi Uber que han tenido una gran acogida por los clientes, fundamentalmente por la facilidad de elección, confort y precio, teniendo como consecuencia en las empresas turísticas la disminución de ventas y reducción de personal, situaciones problemáticas en la empresa que obliga al gerente entrar en acción demostrando sus habilidades gerenciales y su actitud de mejora.

Para comprender conceptualmente las habilidades gerenciales se revisaron diferentes fuentes, Mosley, Megginson y Pietri (2005) quienes agrupan las habilidades en: a) Habilidades personales, es decir, las habilidades técnicas, humanas y conceptuales; b) Habilidades interpersonales, que comprenden asertividad, empatía, manejo de relaciones sociales, solución de problemas, colaboración y comunicación; c) Habilidades de grupo, como son las participativas, comunicativas y colaborativas.

Whetten y Cameron (2011) plantearon diez habilidades gerenciales agrupándose en: a) Personales, autoconocimiento, manejo del estrés, del tiempo y solución a los problemas; b) Interpersonales, como el manejo de conflictos, motivación, comunicación y liderazgo; c) Grupales, que son el empowerment, dirección de cambio y el trabajo en equipo.

Puga y Martínez (2008 en Rebolledo, Flores, Reyes y Flores, 2015) describen que "las cinco habilidades mínimas que requieren poseer los gerentes o directivos de los nuevos tiempos 
y que son: 1) Orientado al conocimiento; 2) Liderazgo, 3) Habilidad para comunicar, 4) Valores éticos, 5) Capacidad para construir equipos de trabajo" (p. 59).

Reh (2009) agrupa las habilidades directivas según la responsabilidad: 1er nivel habilidades básicas, que comprende el proceso administrativo; 2do nivel - habilidades en el trato del trabajador, que implican la motivación, capacitación, entrenamiento y el fomento de la participación; 3er nivel - habilidades de implicación personal, referidas a la efectividad y la gestión del tiempo; 4to nivel - liderazgo, que comprende la gestión de la organización.

Katz (citado en Aburto y Bonales, 2011; Lopes y otros, 2010) identificó tres habilidades gerenciales:

1. Habilidades técnicas, comprende conocimiento especializado, competencias en un área del saber y proceso.

2. Habilidades de trato personal y de trabajo en equipo, se refiere a la capacidad para trabajar con otras personas en el trabajo, relación interpersonal y grupal, comunicación, motivación, coordinación, liderazgo y resolución de conflictos.

3. Habilidades conceptuales, comprende la capacidad para pensar, aplicación conceptos, teorías, atención de situaciones abstractas y complicadas.

El desempeño de las habilidades gerenciales tiene relación directa con el nivel jerárquico (estratégico, táctico y operacional), los gerentes o la alta gerencia despliegan con mayor amplitud la habilidad conceptual, porque tienen una visión holística de la organización y su relación con el entorno; mientras que el nivel operacional u operativo está vinculado a las actividades cotidianas (ejecución de procesos) razón por la cual desarrollan predominantemente la habilidad técnica; sin embargo, la habilidad humana es importante a todo nivel organizacional en vista que permite la fluidez de la comunicación, las relaciones interpersonales, la negociación, la solución de problemas, entre otras.

Para el estudio se revisaron diferentes artículos que median las habilidades gerenciales; Paredes, Mendoza y Partida (2017) establecieron las siguientes dimensiones: Manejo del estrés laboral solución de problemas, motivación laboral, liderazgo e innovación; el cuestionario fue aplicado a gerentes que tenían entre 11 a 250 empleados, siendo la muestra de 81 encuestados, demostrando que las habilidades de liderazgo e innovación influyen en el proceso administrativo.

Leyva, Espejel y Cavazos (2017) determinaron que las habilidades gerenciales estarían compuestas por: a) H. operatividad y de gestión; b) H. Administración estratégica; c) H. planeación estratégica; d) H. globalización; e) H. recursos humanos, el instrumento se aplicó a una muestra de 108 empresarios, exponiendo que la visión de globalización (toma de decisiones, confianza, negociación, análisis financiero, métodos para evaluar proyectos, procesos y productos) tiene incidencia directa en la competitividad empresarial.

Mostafa, Habib, Farzad y Nahid (2012) diseñaron el cuestionario compuesto por 44 preguntas distribuidos en tres dimensiones como fueron: conceptual (15 preguntas), humanas (17 preguntas) y técnicas (12 preguntas), identificando que la habilidad humana es la de mayor importancia, seguida por la habilidad conceptual y finalmente la técnica. 


\section{Método}

El estudio se realizó en las empresas vinculadas al sector turismo ubicadas en el distrito de Miraflores en el periodo de agosto a diciembre de 2018, la muestra de estudio estuvo constituida por 50 gerentes.

La investigación se ejecutó bajo el enfoque cuantitativo, diseño descriptivo simple, transversal y de proceso desarrollado en cuatro etapas, porque al crear un instrumento se tuvo que realizar las modificaciones según los resultados del valor KMO y Barlett (Hernández, Fernández y Baptista, 2014).

\section{Primera etapa}

El instrumento fue diseñado en tres dimensiones en concordancia con lo expuesto por Katz: a) Habilidades técnicas, b) habilidades de trato personal y c) habilidades conceptuales compuesto por 16, 21 y 12 ítems respectivamente, la escala de medición fue de Likert: $1=$ Nunca, $2=$ Casi nunca, $3=$ A veces, $4=$ Casi siempre, $5=$ Siempre. El puntaje mínimo del instrumento fue de 49 puntos y el máximo de 245; para la estandarización se aplicó el cuestionario a una muestra de 50 gerentes del sector turismo y, al realizar el procesamiento estadístico a través del análisis factorial exploratorio, el valor de la prueba de KMO y Barlett resultó ser menor a .8 y el nivel de significancia superior a .05 , razón por la cual se realizó la revisión teórica y se replantearon los ítems, es así que se eliminaron las preguntas 2, 34 y 35 luego del análisis de Correlación de anti-imagen.

\section{Segunda etapa}

El instrumento replanteado fue aplicado a la muestra de 50 gerentes del sector turismo, el mismo que estuvo constituido por 45 ítems. Al realizar el análisis factorial no se logró satisfacer los valores de KMO, Barlett y, según el análisis de correlación, se suprimieron los ítems: 2, 5 , $18,20,22$ y 28, reduciéndose el instrumento a 39 preguntas y ameritando una siguiente aplicación a la muestra de estudio.

\section{Tercera etapa}

El instrumento constituido por 39 ítems fue aplicado por tercera vez a la muestra de estudio, donde luego del análisis, se suprimieron los ítems 12, 13, 18, 20, 21, 24, 26, 27; reduciéndose a 31 ítems y ameritando una cuarta aplicación.

\section{Cuarta etapa}

El instrumento constituido por 31 ítems se aplicó a la muestra de estudio, donde se obtuvo el valor de KMO y Barlett $(.875, \mathrm{p}<0.05)$ aceptable tal como se aprecia en la tabla 1. 
Tabla 1

Prueba de KMO y Barlett aplicado al cuestionario de habilidades gerenciales en el sector turismo

\begin{tabular}{lrr}
\hline & Prueba estadística & Valor \\
\hline Medida de adecuación muestral de Kaiser-Meyer-Olkin. & &, 875 \\
& Chi-cuadrado aproximado & 1232,394 \\
Prueba de esfericidad de Bartlett & Gl & 465 \\
& Sig. &, 000 \\
\hline
\end{tabular}

Nota: Resultado obtenido luego de la cuarta aplicación del cuestionario

\section{Resultados de fiabilidad}

El instrumento se sometió a la prueba de confiabilidad de Alfa de Cronbach, el mismo que tiene como objetivo calcular el promedio de correlaciones entre los ítems; también se optó por la prueba de Dos mitades de Guttman porque el objetivo fue medir la correlación entre los dos grupos de ítems (Kerlinger y Lee, 2002). En la tabla 2 se presentan los valores de Alfa de Cronbach superior al valor 0.7 (Celina y Campo, 2005) demostrando así la consistencia interna del instrumento, del mismo modo los resultados de la prueba de las dos mitades de Guttman arrojaron valores superiores a .80 , por lo tanto, el instrumento es confiable.

Tabla 2

Pruebas de confiabilidad del cuestionario de habilidades gerenciales en el sector turismo

\begin{tabular}{|c|c|c|c|c|c|}
\hline \multirow[b]{2}{*}{ Dimensión } & \multirow[b]{2}{*}{ Ítems } & \multirow[b]{2}{*}{$\begin{array}{r}\text { Alfa de } \\
\text { Cronbach }\end{array}$} & \multicolumn{2}{|c|}{$\begin{array}{c}\text { Análisis parcial para la prueba de Dos } \\
\text { mitades de Gutmann }\end{array}$} & \multirow[b]{2}{*}{$\begin{array}{l}\text { Dos mitades de } \\
\text { Guttman }\end{array}$} \\
\hline & & & $\begin{array}{r}\text { Parte } 1 \\
\text { Alfa de } \\
\text { Cronbach }\end{array}$ & $\begin{array}{r}\text { Parte } 2 \\
\text { Alfa de } \\
\text { Cronbach }\end{array}$ & \\
\hline $\begin{array}{l}\text { Habilidades } \\
\text { técnicas }\end{array}$ & $1-10$ & .910 & .831 & .837 & .912 \\
\hline $\begin{array}{l}\text { Habilidades de trato } \\
\text { personal }\end{array}$ & $\begin{array}{l}11- \\
21\end{array}$ & .915 & .886 & .884 & .885 \\
\hline $\begin{array}{l}\text { Habilidades } \\
\text { conceptuales }\end{array}$ & $\begin{array}{l}22- \\
31\end{array}$ & .922 & .857 & .869 & .900 \\
\hline $\begin{array}{l}\text { Habilidades } \\
\text { gerenciales }\end{array}$ & $1-31$ & .968 & .947 & .940 & .918 \\
\hline
\end{tabular}

\section{Resultados}

La muestra de estudio estuvo constituida por 50 gerentes, de los cuáles el 54\% (27) fueron del género masculino, el 46\% (23) del género femenino; la edad mínima fue de 37 , máxima de 65, promedio 51, la desviación estándar de 8 años. La actividad económica predominante fue restaurante y transporte $22 \%(11-11)$ en cada uno de ellos, seguido por catering 20\% (10), hotel y agencia de viajes $18 \%(9-9)$. En la tabla 3 se presenta la tabla de contingencia entre la actividad económica y el género, resultando que predomina el género masculino (54\%) y se encuentran concentrados en la actividad económica de transporte y catering, mientras que en género femenino (46\%) se concentran en la actividad de restaurante $14 \%(7)$. 
Tabla 3

Tabla de contingencia de la actividad económica y género de la muestra de estudio

\begin{tabular}{|c|c|c|c|c|c|}
\hline & & & & & Total \\
\hline & & & Masculino & Femenino & \\
\hline & & Recuento & 4 & 7 & 11 \\
\hline & Restaurante & $\%$ del total & $8,0 \%$ & $14,0 \%$ & $22,0 \%$ \\
\hline & & Recuento & 3 & 6 & 9 \\
\hline & Hotel & $\%$ del total & $6,0 \%$ & $12,0 \%$ & $18,0 \%$ \\
\hline Actividad & A rancio do & Recuento & 6 & 3 & 9 \\
\hline Económica & Agencia de viajes & $\%$ del total & $12,0 \%$ & $6,0 \%$ & $18,0 \%$ \\
\hline & Trancnorte & Recuento & 7 & 4 & 11 \\
\hline & 11ansporte & $\%$ del total & $14,0 \%$ & $8,0 \%$ & $22,0 \%$ \\
\hline & Cotoring & Recuento & 7 & 3 & 10 \\
\hline & Catering & $\%$ del total & $14,0 \%$ & $6,0 \%$ & $20,0 \%$ \\
\hline Total & & Recuento & 27 & 23 & 50 \\
\hline lotal & & $\%$ del total & $54,0 \%$ & $46,0 \%$ & $100,0 \%$ \\
\hline
\end{tabular}

Después de realizar el análisis factorial se identificó que el modelo para medir las habilidades gerenciales estaría constituido por las siguientes dimensiones:

1. Dimensión 1 habilidades técnicas $(\mathrm{HT})$ integrada por 10 ítems, distribuidos en tres indicadores: a) Software, ítems 1 al 4; b) Idioma, ítems 5 al 6; c) Negociación y decisiones, ítems 7 al 10.

2. Dimensión 2 habilidades de trato personal (HP) constituida por 11 ítems, distribuidos en tres indicadores: a) Autocontrol, ítems 11 al 14; b) Autoconfianza, ítems 15 al 16; c) Equilibrio emocional, ítems 17 al 18; d) Empatía, ítems 19 al 21.

3. Dimensión 3 habilidades conceptuales (HC) conformada por 10 ítems, distribuidos en dos indicadores: a) Desarrollo de nuevos conceptos, ítems 22 al 26; b) Resolución de problemas, ítems 27 al 31; como se esquematiza en la figura 1.

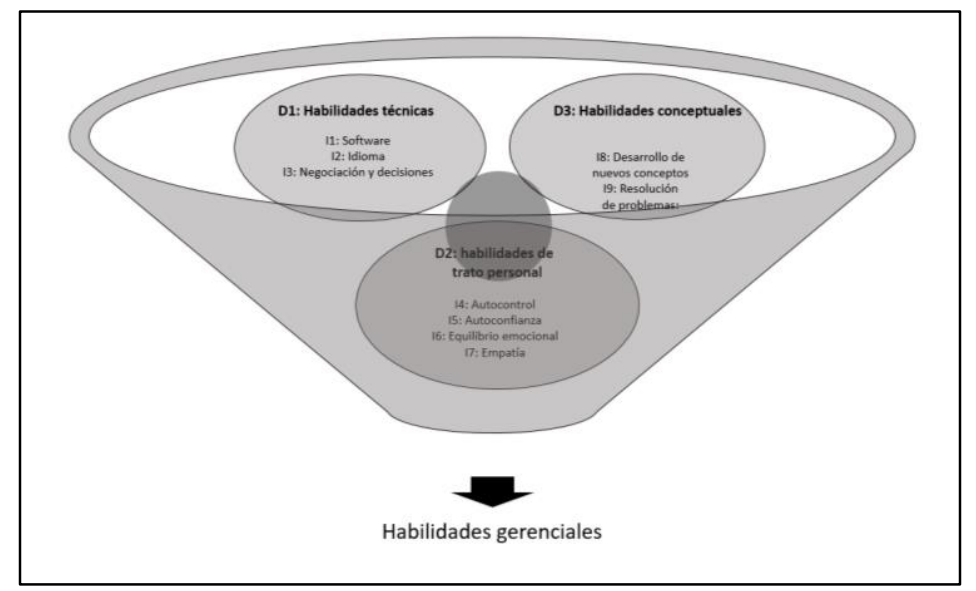

Figura 1. Modelo de las habilidades gerenciales.

Fuente: Elaboración propia.

Luego se realizó el análisis factorial con el método de análisis de componentes principales, se identificó que los componentes del 1 al 6 obtuvieron auto valores iniciales superiores a 1, acumulando una varianza total explicada de $72.647 \%$, es decir estos seis 
elementos representan al problema o permiten medir a la variable en un $72.647 \%$ como se aprecia en la tabla 4 y figura 1 .

Tabla 4

Matriz de componentes rotados $(n=50)$

\begin{tabular}{|c|c|c|c|c|c|c|}
\hline \multirow{2}{*}{ Componente } & \multicolumn{3}{|c|}{ Auto valores iniciales } & \multicolumn{3}{|c|}{$\begin{array}{c}\text { Sumas de las saturaciones al cuadrado de la } \\
\text { extracción }\end{array}$} \\
\hline & Total & $\begin{array}{c}\% \text { de la } \\
\text { varianza }\end{array}$ & $\begin{array}{c}\% \\
\text { acumulado }\end{array}$ & Total & $\%$ de la varianza & $\%$ acumulado \\
\hline 1 & 15,837 & 51,086 & 51,086 & 15,837 & 51,086 & 51,086 \\
\hline 2 & 1,796 & 5,795 & 56,881 & 1,796 & 5,795 & 56,881 \\
\hline 3 & 1,515 & 4,886 & 61,767 & 1,515 & 4,886 & 61,767 \\
\hline 4 & 1,222 & 3,940 & 65,707 & 1,222 & 3,940 & 65,707 \\
\hline 5 & 1,098 & 3,543 & 69,250 & 1,098 & 3,543 & 69,250 \\
\hline 6 & 1,053 & 3,398 & 72,647 & 1,053 & 3,398 & 72,647 \\
\hline 7 & ,980 & 3,161 & 75,808 & & & \\
\hline 8 & ,928 & 2,994 & 78,802 & & & \\
\hline 9 &, 775 & 2,500 & 81,303 & & & \\
\hline 10 &, 681 & 2,198 & 83,500 & & & \\
\hline 11 &, 645 & 2,079 & 85,580 & & & \\
\hline 12 &, 551 & 1,778 & 87,358 & & & \\
\hline 13 & ,494 & 1,595 & 88,953 & & & \\
\hline 14 & ,447 & 1,441 & 90,394 & & & \\
\hline 15 & ,416 & 1,341 & 91,735 & & & \\
\hline 16 &, 340 & 1,096 & 92,831 & & & \\
\hline 17 & ,308 & ,995 & 93,826 & & & \\
\hline 18 & ,292 & ,943 & 94,769 & & & \\
\hline 19 &, 241 & ,778 & 95,548 & & & \\
\hline 20 &, 221 & ,713 & 96,261 & & & \\
\hline 21 & ,198 & ,639 & 96,899 & & & \\
\hline 22 &, 181 &, 585 & 97,485 & & & \\
\hline 23 &, 152 & ,492 & 97,976 & & & \\
\hline 24 & ,126 & ,406 & 98,382 & & & \\
\hline 25 & ,109 &, 353 & 98,735 & & & \\
\hline 26 &, 100 &, 324 & 99,059 & & & \\
\hline 27 & ,076 & ,244 & 99,303 & & & \\
\hline 28 & ,066 & ,213 & 99,515 & & & \\
\hline 29 & ,058 & , 189 & 99,704 & & & \\
\hline 30 &, 052 & ,166 & 99,870 & & & \\
\hline 31 & ,040 &, 130 & 100,000 & & & \\
\hline
\end{tabular}

Método de extracción: Análisis de Componentes principales. 


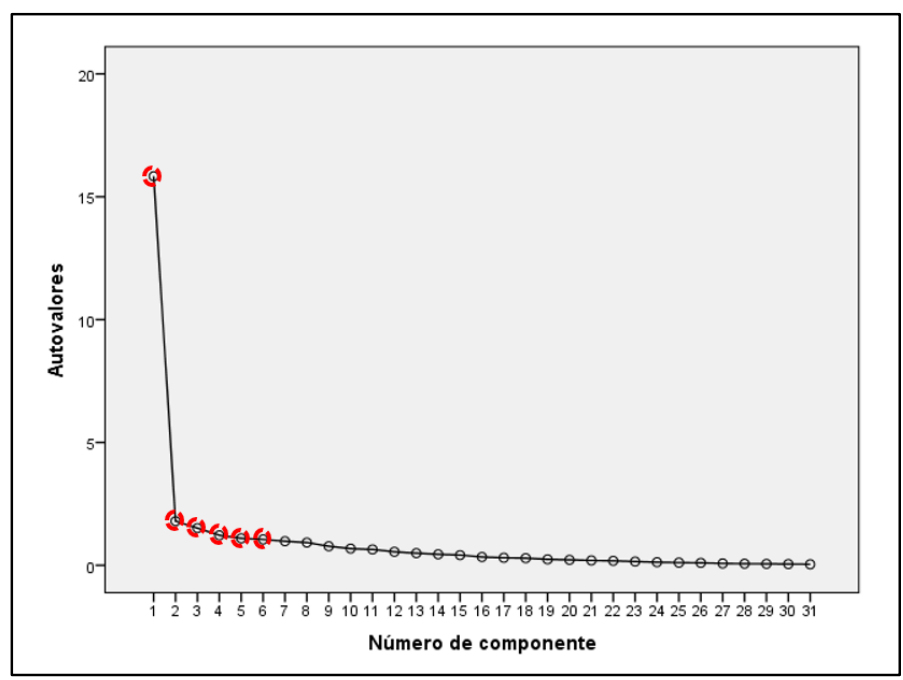

Figura 2. Gráfico de sedimentación

Se observa en la tabla 5 la matriz de componentes rotados, el objetivo fue analizar la correlación del ítem con el factor, determinándose que del ítem 1 al 10 corresponde a las habilidades técnicas, del 11 al 21 a las habilidades personales y del 22 al 31 a las habilidades conceptuales.

Tabla 5

Matriz de componentes rotados $(n=50)$

\begin{tabular}{|c|c|c|c|c|}
\hline \multirow{2}{*}{ Nro. } & \multirow{2}{*}{ Ítem } & \multicolumn{3}{|c|}{ Componente } \\
\hline & & HT & HP & $\mathrm{HC}$ \\
\hline 1. & Ud. se capacita constantemente en el uso de software & .618 & .095 & .482 \\
\hline 2. & Ud. utiliza Ms Excel para la toma de decisiones & .731 & .257 & .130 \\
\hline 3. & Ud. utiliza Prezi, PowToon u otra herramienta para sus presentaciones & .815 & .127 & .141 \\
\hline 4. & Ud. utiliza software de diseño gráfico para la documentación & .567 & .353 & .431 \\
\hline 5. & Ud. habla otro idioma para sus negociaciones & .636 & .333 & 196 \\
\hline 6. & Ud. escribe en otro idioma para sus negociaciones & .617 & .451 & .332 \\
\hline 7. & Ud. cuando va a negociar recopila información sobre su contraparte & .492 & .329 & .251 \\
\hline 8. & Ud. averigua antes de la negociación lo que ofrece la competencia & .707 & .162 & .318 \\
\hline 9. & Ud. soluciona conflictos previa negociación & .535 & .341 & .385 \\
\hline & $\begin{array}{l}\text { Ud. para tomar decisiones usa alguna herramienta como: Árbol de Problemas, } \\
\text { Ishikawa, Pareto para la toma de decisiones }\end{array}$ & .575 & .424 & .344 \\
\hline & Ud. controla sus impulsos emocionales & .303 & .506 & .494 \\
\hline 12. & Ud. controla el estrés en su desempeño laboral & .307 & .660 & .438 \\
\hline & $\begin{array}{l}\text { Ud. realiza sus actividades con normalidad independientemente de sus } \\
\text { emociones }\end{array}$ & .601 & .624 & .083 \\
\hline 14. & Ud. trata de mantener la calma a pesar de la situación & .461 & .469 & .404 \\
\hline 15. & Ud. toma las decisiones con total seguridad & .412 & .587 & .347 \\
\hline 16. & Ud. tiene plena confianza en sus capacidades & .462 & .543 & .161 \\
\hline 17. & Ud. tiene paciencia con las personas que tardan en realizar su tarea & .178 & .548 & .511 \\
\hline 18. & Ud. se deja influenciar por los cambios de emociones de su entorno & .235 & .508 & .388 \\
\hline 19. & Ud. brinda confianza para que sus colaboradores dialoguen con su persona & .371 & .732 & 138 \\
\hline 20. & Ud. trata de entender a sus colaboradores ante una situación & .251 & .709 & .266 \\
\hline 21. & Ud. considera los sentimientos de las personas para la toma de decisiones & .194 & .669 & .203 \\
\hline 22. & Ud. desarrolla nuevos productos para lograr el posicionamiento de la empresa & .368 & .189 & .663 \\
\hline 23. & Ud. desarrolla nuevos productos para lograr rentabilidad & .381 & .294 & .594 \\
\hline 24. & Ud. ha implementado en el último año nuevos productos para la empresa & .226 & .078 & .816 \\
\hline
\end{tabular}




\begin{tabular}{rlrrr}
\hline \multirow{2}{*}{ Nro. } & \multicolumn{1}{c}{ Ítem } & \multicolumn{3}{c}{ Componente } \\
& & HT & HP & HC \\
\hline 25. & Ud. ha implementado en los últimos 5 años nuevos productos para la empresa & .321 & .281 & .621 \\
26. & Ud. está actualmente pensando en nuevos productos & .454 & .125 & .681 \\
27. & Ud. evalúa varias alternativas para la solución de los problemas & .240 & .328 & .707 \\
28. & Ud. ante un problema asume una actitud constructiva y aprende de ellos & .378 & .181 & .676 \\
29. & Ud. para solucionar un problema considera las sugerencias de sus & .282 & .391 & .533 \\
colaboradores & Ud. ante un problema de su personal procura solucionarlo con prontitud & .355 & .213 & .728 \\
31. & Ud. ante un problema asume una actitud drástica & .506 & .076 & .673 \\
\hline
\end{tabular}

Método de extracción: Análisis de componentes principales.

Método de rotación: Normalización Varimax con Kaiser.

a. La rotación ha convergido en 8 iteraciones.

\section{Discusión}

El cuestionario HGT se ha construido según las dimensiones expuestas por Katz, identificando tres dimensiones: a) habilidades técnicas, b) habilidades de trato de personas, c) habilidades conceptuales. Para la estandarización se aplicó el instrumento a una muestra de 50 gerentes o directivos de las empresas vinculadas a la actividad turística, obteniendo en primera instancia valores no aceptables, luego se realizaron las modificaciones respectivas, en la cuarta aplicación se logró obtener KMO = .875 y la Prueba de esfericidad de Barlett $p=.000$, determinando así que el instrumento es aceptable.

También se aplicó la prueba de confiabilidad de Alfa de Cronbach en vista que la escala de medición fue Likert, obteniendo de manera general .968, mientras que en las dimensiones los valores fueron de .910, .915 y .922; así también se aplicó la prueba de Dos mitades de Guttman, logrando de manera general .918 y las dimensiones $.912, .88, .900$, por lo tanto, se demostró estadísticamente que el instrumento posee consistencia interna.

Mediante el análisis de matriz de componentes rotados se evidenció que los ítems del 1 al 6 explican a las habilidades gerenciales en el sector turismo en un $72.647 \%$ y que el $27.353 \%$ es explicado por otros factores que no fueron parte del estudio, las cargas factoriales demostraron que del ítem 1 al 10 corresponden a las habilidades técnicas, mientras que del 11 al 21 permiten medir las habilidades de trato de personas, y los ítems del 22 al 31 comprenden a las habilidades conceptuales.

Es necesario hacer hincapié en que se ha seguido rigurosamente el desarrollo metodológico planeado, además de cumplir con los requisitos teóricos, confiabilidad y análisis factorial para la estandarización.

\section{Conclusiones}

Los gerentes de las empresas cada vez se enfrentan a mayores desafíos organizacionales, son conscientes que sus decisiones tendrán consecuencias, esta situación genera preocupación, stress, falta de auto control y equilibrio emocional, haciendo que se desliguen o se quiebre la comunicación con los niveles operativos, afectando directamente al logro de los objetivos organizacionales. 
En tal sentido es necesario que el gerente logre manejar sus emociones y consiga la sinergia de las diferentes habilidades gerenciales, para lograr la integración interna y el compromiso de los colaboradores. Un buen gerente no solo se preocupa por el logro de los objetivos (cuantitativos), sino también busca el crecimiento personal de sus colaboradores, a través de capacitaciones, pasantías, descansos, premios, los mismos que son muy valorados y apreciados.

Una forma para medir las habilidades gerenciales es según las tres habilidades, técnicas, de trato personal y conceptuales sustentadas por Katz, sin embargo, no se descarta otros conceptos como los de Mosley et. al., Whetten y Cameron, Rebolledo et. al., Reh. Sino por el contrario, en un futuro se podría diseñar y aplicar un cuestionario asociando otras perspectivas.

\section{Agradecimientos}

A la Universidad Norbert Wiener por el financiamiento de la investigación a través del Fondo concursable 2018.

To the Norbert Wiener University for the research financial support through de 2018 Fondo Concursable.

\section{Bibliografía}

Aburto, H., \& Bonales, J. (2011). Habilidades directivas: Determinantes en el clima organizacional. investigación y ciencia de la Universidad Autónoma de Aguascalientes, 41-49.

Celina, H., \& Campo, A. (2005). Aproximación al uso del coeficiente alfa de Cronbach. Revista colombiana de psiquiatría, XXXIV(004), 572-580.

Hernández, R., Fernández, C., \& Baptista, M. (2014). Metodología de la investigación. México: McGraw-Hill.

Hernández, R., Fernández, C., \& Baptista, P. (2010). Metodología de la investigación. México: Mc Graw Hill.

Katz, R. (1974). Skills of an Effective Administrator. Harvard Business Review(52), 90-102.

Kerlinger, F., \& Lee, H. (2002). Investigación del comportamiento. México: Mc Graw Hill.

Leyva, A., Espejel, J., \& Cavazos, J. (2017). Habilidades gerenciales como estrategia de competitividad empresarial en las pequeñas y medianas empresas (Pymes). RPE, 4(1), 722.

Lopes, M., Pereira, F., Leonardi, J., Lopes, L., Fernandes, S., \& Wihelm, P. (2010). Análise da relação entre aptidões cerebrais e competências gerenciais: o caso de uma empresa têxtil. Gest. Prod., 17(1), 123-136.

Maximiano, A. (2007). Introdução à administração. Sao Paulo: Atlas.

Mosley, D., Megginson, L., \& Pietri, P. (2005). La práctica del empowerment, desarrollo de equipo de trabajo y su motivación. México: Editorial Thomson.

Mostafa, A., Habib, H., Farzad, Q., \& Nahid, J. (2012). Prioritizing Managerial Skills Based on Katz's Theory in Physical Education Offices of Universities in Iran. World Applied Sciences Journal, 20(3), 388-394. doi:10.5829/idosi.wasj.2012.20.03.1169 
Paredes, D., Mendoza, J., \& Partida, A. (2017). Validación del instrumento para medir el impacto de las habilidades gerenciales del capital humano como estrategia de buen funcionamiento del proceso administrativo de las Pymes al comercio por mayor ubicadas en ciudad Obregón Sonora, México. TEACS, 9(20), 25-43.

Ramírez, J. (2018). Las habilidades directivas una condición para una ejecución eficaz. Revista Investigación y Negocios, 11(17), 23-29.

Rebolledo, A., Flores, J., Reyes, T., \& Flores, J. (2015). Investigación en las ciencias de la gestión, innovación, competitividad y tecnologías de la información. México: Competitive Press S.A. de CV.

Reh, F. (2009). The Management Skills Pyramid Management. Chicago: Harthor Edition.

Reyes, A. (2015). Administración de empresas: teoría y práctica. México: Editorial Limusa S.A.

Robbins, S., \& Coulter, M. (2005). Administración. México: Pearson Educación.

Whetten, D., \& Cameron, K. (2011). Desarrollo de habilidades directivas. México: Pearson Educación de México. 


\section{Apéndice 1: cuestionario para medir las habilidades gerenciales}

Estimado gerente:

El presente cuestionario tiene como objetivo recopilar los datos de las habilidades gerenciales que usted practica en su desempeño profesional. En tal sentido se solicita su colaboración seria y responsable para contestar las preguntas, cabe precisar que no existe respuesta buena ni mala, todas son válidas para el estudio.

Nro. Ítem

1. Ud. Se capacita constantemente en el uso de software

2. Ud. Utiliza Ms Excel para la toma de decisiones

3. Ud. Utiliza Prezi, Pow Town u otra herramienta para sus presentaciones

4. Ud. Utiliza software de diseño gráfico para la documentación

5. Ud. Habla otro idioma para sus negociaciones

6. Ud. Escribe en otro idioma para sus negociaciones

7. Ud. Cuando va a negociar recopila información sobre su contraparte

8. Ud. Averigua antes de la negociación lo que ofrece la competencia

9. Ud. Soluciona conflictos previa negociación

10. Ud. Para tomar decisiones usa alguna herramienta como: Árbol de problemas,

10. Ishikawa, Pareto para la toma de decisiones

11. Ud. Controla sus impulsos emocionales

12. Ud. Controla el estrés en su desempeño laboral

13. Ud. Realiza sus actividades con normalidad independientemente de sus emociones

14. Ud. Trata de mantener la calma a pesar de la situación

15. Ud. Toma las decisiones con total seguridad

16. Ud. Tiene plena confianza en sus capacidades

17. Ud. Tiene paciencia con las personas que tardan en realizar su tarea

18. Ud. Se deja influencia por los cambios de emociones de su entorno

19. Ud. Brinda confianza para que sus colaboradores dialoguen con su persona

20. Ud. Trata de entender a sus colaboradores ante una situación

21. Ud. Considera los sentimientos de las personas para la toma de decisiones

22. Ud. Desarrolla nuevos productos para lograr el posicionamiento de la empresa

23. Ud. Desarrolla nuevos productos para lograr rentabilidad

24. Ud. Ha implementado en el último año nuevos productos para la empresa

25. Ud. Ha implementado en los últimos 5 años nuevos productos para la empresa

26. Ud. Esta actualmente pensando en nuevos productos

27. Ud. Evalúa varias alternativas para la solución de los problemas

28. Ud. Ante un problema asume una actitud constructiva y aprende de ellos

29. Ud. Para solucionar un problema considera las sugerencias de sus colaboradores

30. Ud. Ante un problema de su personal procura solucionarlo con prontitud

31. Ud. Ante un problema asume una actitud drástica 